\title{
Superficial Temporal Artery Pseudoaneurysm following a Traumatic Haircut
}

\author{
Matthew L Carnevale ${ }^{1}$, John Phair ${ }^{2}$, Eric B Trestman², Gregory Dickinson ${ }^{3}$ and Michael J Vitti \\ ${ }^{1}$ Albert Einstein College of Medicine, USA \\ ${ }^{2}$ Department of Vascular Surgery, Montefiore Medical Center, USA \\ ${ }^{3}$ Department of Pathology, Montefiore Medical Center, USA

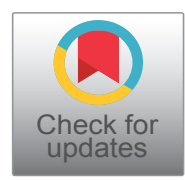

*Corresponding author: Dr. John Phair, MD, Department of Vascular Surgery, Montefiore Medical Center, 111 East $210^{\text {th }}$ Street, Bronx, NY 10467, USA, Tel: 718-920-4321, Fax: 424-251-9337,E-mail: jphair@montefiore.org

\begin{abstract}
Superficial temporal artery pseudoaneurysms are well known to occur following blunt trauma to the forehead, but their manifestation after penetrating trauma is considerably less often reported. A middle-aged male presented with a pulsatile mass on his forehead that had been growing slowly. He reports being accidentally cut by his barber in that location 2-3 months prior. This was determined to be a superficial temporal artery aneurysm by physical diagnosis. The aneurysm was surgically resected, and the patient recovered uneventfully. Owing to their relative exposure compared to other arteries, the superficial temporal artery is particularly at risk of pseudoaneurysm formation secondary to trauma. These lesions can be successfully treated with simple ligation and excision.
\end{abstract}

\section{Introduction}

The superficial temporal artery, one of the terminal branches of the external carotid artery is tasked with supplying blood to the anterior and lateral scalp. Due to its relatively superficial location it is particularly vulnerable to trauma of the head. Blunt head trauma has been shown to be capable of causing pseudoaneurysm of the superficial temporal artery in the following few weeks [1]. Roughly 200 cases have been described so far and the vast majority is associated with blunt rather than penetrating trauma [2]. Blunt trauma to the side of the head results in a shearing and crushing force on the superficial temporal artery between the skin and the bony ridge that acts as the origin of the temporalis muscle [3]. Blunt trauma to the same area is well known to cause aneurysms but penetrating trauma as a cause of superficial temporal artery aneurysms has not been well documented in the literature. Here we present a patient who developed an aneurysm after a penetrating injury to the scalp.

\section{Case Report}

A 59-year-old male with no significant past medical or surgical history presented with a focal swelling on the right side of his forehead which he noticed 2

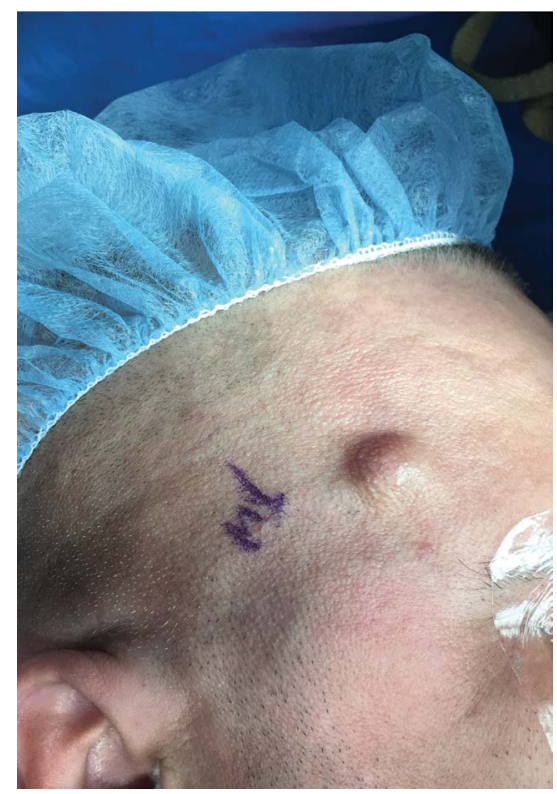

Figure 1: Pulsatile mass measuring $1.5 \mathrm{~cm}$ on anterolateral forehead. 


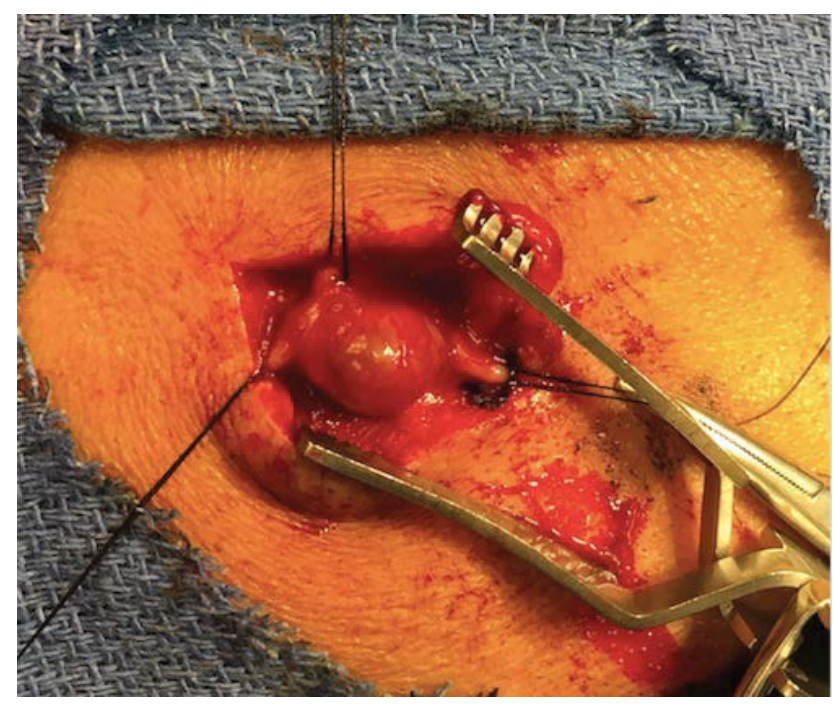

Figure 2: Surgical image demonstrating superficial temporal artery aneurysm.

months prior. He reported that the mass had been slowly enlarging during that time. He reported a minor accidental scissor injury by his barber in the same area prior to the appearance of the mass. Physical examination revealed a smooth mass that was pulsatile, non-fluctuant, non-erythematous, non-tender and measured 1.5 $\mathrm{cm}$ in diameter (Figure 1). The mass became non-pulsatile with compression of the superficial temporal artery, proximal to the area of swelling. The patient was treated with surgical resection of the mass which turned out to be a pseudoaneurysm of the superficial temporal artery (Figure 2). Segments of the superficial temporal artery proximal and distal to the aneurysm were ligated before the mass was excised. The patient recovered from the procedure uneventfully. Pathologic examination revealed a pseudoaneurysm with an organizing transmural thrombus that was partially occluding the vessel lumen (Figure 3).

\section{Discussion}

Most pseudoaneurysms of the superficial temporal artery present as painless, pulsatile masses that may be associated with a palpable thrill. While up to $95 \%$ of superficial temporal artery pseudoaneurysms are associated with antecedent trauma, the vast majority are secondary to blunt injuries [4]. This case demonstrates that penetrating trauma can be a cause of superficial temporal artery pseudoaneurysms. Other etiologies include spontaneous superficial temporal artery aneurysms secondary to atherosclerosis and congenital arterial wall weakness [5].

The differential diagnosis of a pulsatile mass on the anterolateral aspect of the scalp includes vascular tumors, arteriovenous fistulae, and aneurysms of adjacent arteries. Diagnosis is made primarily by history of trauma in the previous 2-8 weeks and physical findings including a solitary, pulsatile, non-fluctuant mass in the anterolateral forehead that may be expansile [6]. Palpation of the lesion with simultaneous occlusion of the proximal superficial temporal artery should result in loss

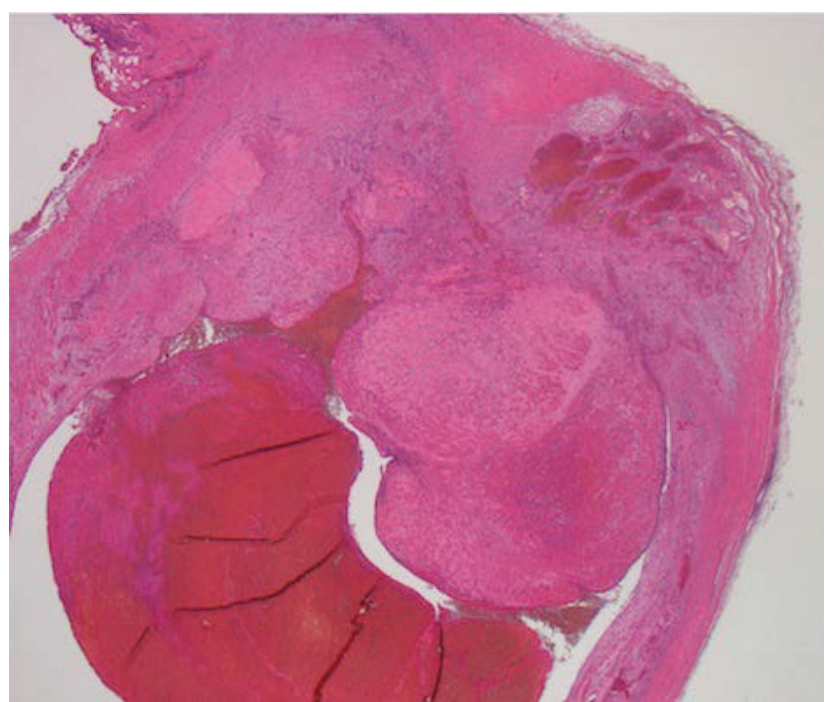

Figure 3: Photomicrograph of an aneurysm of the superficial temporal artery. An organizing transmural thrombus is observed with a portion partially occluding the lumen.

of the palpable pulses in the mass [7]. Duplex sonography can be used to confirm the diagnosis of superficial temporal artery aneurysms [8]. Additional imaging modalities such as CTA/MRA can also be used to diagnose superficial temporal artery aneurysms.

Elective surgical resection of the superficial temporal artery aneurysm is the treatment modality of choice. While conservative management with hematoma compression has been postulated, it has been shown to be ineffective. Endovascular treatment such as embolization as well as thrombin injection have been investigated but due to cosmetic reasons are reserved for patients who are not surgical candidates [2]. Dissection of the scalp to reveal the lesion, ligation of proximal and distal vessels, followed by complete excision of the aneurysm is the treatment of choice [6]. The superficial temporal artery anastomoses with the supraorbital artery, a branch of the ophthalmic artery. Due to this anatomical characteristic, patients with occluded ipsilateral internal carotid arteries should not undergo ligation, of the superficial temporal artery aneurysm as this may compromise cerebral blood flow throw the ophthalmic artery.

\section{Funding}

This work was not funded by and received no support from any outside sources.

All the above-mentioned authors contributed equally to the work.

\section{References}

1. DeSanti $L$ (1884) Aneurysms of the temporal region. Arch Gen Med 154: 543-679.

2. van Uden DJ, Truijers M, Schipper EE, Zeebregts CJ, Reijnen MM (2013) Superficial temporal artery aneurysm: Diagnosis and treatment options. Head Neck 35: 608-614.

3. Harris KA, Walker PM, Hardacre GA (1983) Post-traumatic aneurysms of the superficial temporal artery. Can Fam Physician 29: 1001-1003. 
4. Parbhu Keshini C, Brian S Biesman (2008) Traumatic pseudoaneurysm of the superficial temporal artery. Ophthalmic Plast Reconstr Surg 24: 501-503.

5. Grasso RF, Quattrocchi CC, Crucitti P, Carboni G, Coppola R, et al. (2007) Superficial temporal artery pseudoaneurysm: A conservative approach in a critically ill patient. Cardiovasc Intervent Radiol 30: 286-288.

6. Shenoy SN, Raja A (2003) Traumatic superficial temporal artery aneurysm: Case report. Neurology India 51: 537-538.

7. Sawyer O, Staruch R, Ellabban M (2014) Post traumatic superficial temporal artery aneurysm: Highlighting the importance in history and examination. Eplasty 14: 48.

8. Stapleton CJ, Fusco MR, Thomas AJ, Levy El, Ogilvy CS (2014) Traumatic pseudoaneurysms of the superficial temporal artery: Case series, anatomy, and multidisciplinary treatment considerations. J Clin Neurosci 21: 1529-1532. 